\title{
Mobile Manipulation
}

\section{Vincze, M. Brandstötter}

Online publiziert am 2. September 2020

(c) Springer-Verlag GmbH Austria, ein Teil von Springer Nature 2020

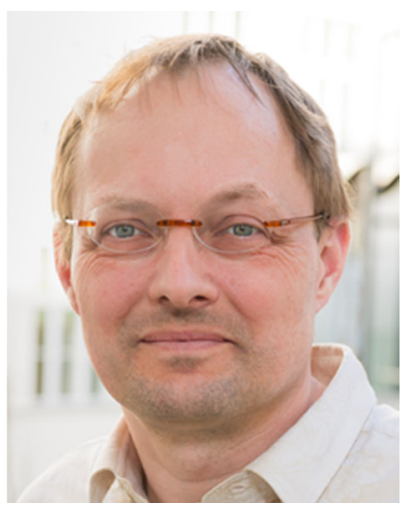

Das Zusammenrücken von Menschen und Maschine prägten die letzten Jahre der angewandten Forschung für Industrie und Service. Diese kontinuierliche Annäherung basiert auf dem besseren gegenseitigen Verständnis, der Simplifizierung der Interaktionsschnittstellen und einer fortlaufend zielorientierteren Systementwicklung. Wird dem Menschen Ao. Univ.-Prof. Dipl.-Ing. Dr.techn. Markus Vincze

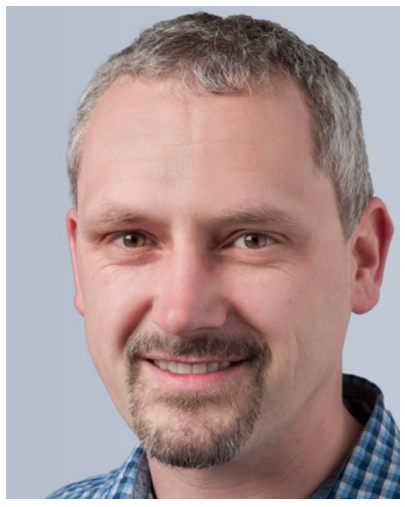

DI Dr. Mathias Brandstötter eine Maschine zur Unterstützung in seiner gewohnten dynamischen Welt bereitgestellt, muss sich diese auf eine aktuell geforderte Arbeitsaufgabe und teils komplexe Umgebungssituationen anpassen können. Das sind jene Eigenschaften, die im Besonderen Robotersysteme auszeichnen. Eine vielversprechende Ausprägung stellen mobile Manipulatoren dar, die Mobilität mit den bekannten Vorzügen von Roboterarmen verbinden. Dadurch entstehen mobile Robotersysteme, die u.a. Bring- und Hol-Tätigkeiten übernehmen, Behälter mit Stückgut befüllen, Material an Fertigungsstationen liefern und auch mit dem Menschen direkt zusammenarbeiten, um Fertigungsaufgaben zu erfüllen. Viele Aspekte dieses herausfordernden Gebiets werden seit vielen Jahren erforscht und deren Resultate rücken einem praxistauglichen Einsatz kontinuierlich näher.

Ziel dieses Sonderheftes ist es, die derzeitigen Möglichkeiten mobiler Manipulatoren aufzuzeigen und darzulegen, welches Potenzial in der Verbindung von Manipulation und Mobilität liegt, denn solche modernen Robotersysteme sind eine unumgängliche Technologie bei der Digitalisierung von Produktions- und Wertschöpfungsprozessen. Die Beiträge umfassen Themen wie die Sicherheit, Systemaspekte, die Konfiguration von Systemen mit mehreren mobilen Manipulatoren, Aufgaben- und Bewegungsplanung, Navigation und die Erkennung aller Gegenstände in Relation zur Umgebung.

Beim Einsatz von mobilen Manipulatoren mit dem Menschen im sogenannten kollaborativen Betrieb operieren Mensch und Maschine in einem gemeinsamen Bereich, ohne dabei durch physische Schutzeinrichtungen getrennt zu sein. Dies stellt hohe Ansprüche an die Robotersicherheit. Der Beitrag von Rathmair et al. („Sichere und zuverlässige mobile Manipulation") präsentiert die normativen Rahmenbedingungen für den sicheren und zuverlässigen industriellen Einsatz von mobilen Manipulatoren. Der koordinierte Einsatz beider Robotersysteme ermöglicht eine Vielzahl neuer Anwendungsszenarien und erhöht maßgeblich das flexible Einsatzpotenzial. Damit einher geht jedoch die aufwändigere und notwendige Risikobeurteilung des Gesamtsystems im Anwendungskontext.

Aufgrund der steigenden Anforderungen an die Flexibilität und Lieferzeiten einer Produktion kommen zunehmend Teams mobiler Robotersysteme zum Einsatz. Die Arbeit von Kohout et al. ( „A multirobot architecture for the RoboCup Logistics League") präsentiert eine Architektur, um die flexible und robuste Ausführung geforderter Produktionsaufgaben mit verschiedenen Systemkonfigurationen zu testen. Die Idee besteht darin, verschiedene Aspekte, wie abstrakte Planung und Zeitplanung, Verfeinerung der Aufgabenausführung und Verhaltenskontrolle, zu trennen und jede Schicht mit geeigneten Bewegungs- und Fehlerbehebungsfunktionen auszustatten. Die Umsetzung wird anhand von on-Demand-Produktbestellungen im Zuge der RoboCup Logistics League evaluiert. Die flexible Planung und Aufgabenzuweisung gepaart mit einer robusten Aufgabenausführung ermöglichte es, komplexere Produkte zuverlässig und zeitgerecht zu realisieren.

Der Beitrag von Sereinig et al. („A review of the challenges in mobile manipulation: systems design and RoboCup challenges - recent developments with a special focus on the RoboCup") untersucht die Herausforderungen dieser Robotersysteme in weniger strukturierten und dynamischen Umgebungen, insbesondere Szenarien mit engem Kontakt zum Menschen, wie sie in der Industrie, in Bereichen der Dienstleistung, Assistenz oder Teleoperation und auch Rehabilitation allgegenwärtig sind. Der Artikel behandelt die Herausforderungen in Bezug auf das Systemdesign, die Regelung und die Sensorik. Diese Problemstellungen werden in den unterschiedlichen Ligen des RoboCup abgebildet und in Aufgaben im Rahmen von RoboCupRescue- sowie der RoboCup@Work-Ligen analysiert. Innovative Sensorik, basierend auch auf neuartigen Konzepten wie z. B. Soft Robotics, speziell auch taktile und Näherungssensorik, stellt eine essenzielle Komponente für die Zukunft kollaborativer Roboter und Assistenz-Roboter dar. Im Bereich der Regelung sind neben klassischen Konzepten auch zunehmend methodische Ansätze basierend auf künstlicher Intelligenz im Einsatz, um Roboter in hochdynamischen Umgebungen zu steuern.

Eine wichtige Aufgabe bei der Planung von Arbeitsschritten und deren Ausführung ist die Reaktion auf Fehler und Störungen. Saveriano und Piater ( tems for monitoring and executing manipulation tasks") stellen ein Modell für die Überwachung und Ausführung von Roboteraufgaben vor, das aus zwei Komponenten besteht: einem rekonfigurier-

Vincze, Markus, Institut für Automatisierungs- und Regelungstechnik, TU Wien Gußhausstraße 27-29, 1040 Wien, Österreich (E-Mail: vincze@acin.tuwien.ac.at); Brandstötter, Mathias, Institut für Robotik und Mechatronik, JOANNEUM RESEARCH Forschungsgesellschaft mbH, Lakeside B13b, 9020 Klagenfurt, Österreich

(E-Mail: mathias.brandstoetter@joanneum.at) 
baren Verhaltensbaum (Reconfigurable Behavior Trees, RBT) und einem energiebasierten Stabilisator für dynamische Systeme (ESDS) RBTs ist die Entscheidungsfunktion auf übergeordneter Ebene und übt durch eine kontinuierliche Überwachung und Aufgabenumschaltung eine Kontrollfunktion auf die niedrige Ebene aus. ESDS lernt aus einer Handvoll Demonstrationen eine flexible und robuste Bewegungsdarstellung, die es ermöglicht, Bewegungsbahnen mit nachgewiesener Konvergenz zu erzeugen. Das vorgeschlagene Modell wird in einem Sortierszenario getestet und zeigt seine Fähigkeit, Störungen während der Ausführung zu behandeln.

Mobile Manipulation ist das Kernstück eines hochflexiblen und autonomen Produktionssystems. Durch vernetzte und roboterbasierte Automatisierung ist eine individuell angepasste Fertigung möglich, wobei mobile Manipulatoren sowohl bei Transportaufgaben als auch bei der Werkstückbereitstellung eine signifikante Rolle spielen. Eine wichtige Funktion ist die Integration in die Industrieumgebung Der Beitrag von Wöber et al. ( $A$ Autonome mobile Robotik in einer digitalisierten Fertigung") präsentiert die Digitale Fabrik der FH Technikum Wien, eine Forschungs- sowie Lehrplattform zur exemplarischen Erprobung neuer Technologien in der digitalen und flexiblen Produktion. Basierend auf einem konkreten und symbolischen Anwendungsfall diskutiert diese Arbeit verschiedene Methoden zur Navigation mobiler Manipulatoren. Es werden Positionierungsgenauigkeiten basierend auf unterschiedlichen Navigationsmethoden sowie Sicherheitsaspekte und Auswirkungen auf die Handhabung von Objekten diskutiert.

Eine wichtige Aufgabe für mobile Manipulatoren ist die Erkennung der Umgebung, um einen sicheren und vorhersehbaren Betrieb zu gewährleisten. Dazu sollte der mobile Manipulator erklären können, was er tut, um direkte Rückmeldungen an die Benut- zer/innen zu geben. Um dieses Ziel zu erreichen, muss er seine Umgebung und die Objekte darin wahrnehmen und auf semantischer Ebene wiedergeben. Der Beitrag von Vincze et al. („Learning, detecting, and grasping objects in real-world settings") gibt einen Überblick der aktuellen Fortschritte zum Erkennen von Objekten und den Relationen in der Umgebung. Mit dem Aufkommen von Farb- und Tiefenkameras (RGB-D), den Fortschritten bei künstlicher Intelligenz (KI) und Deep-Learning-Methoden gelingt es, eine Vielzahl an Objekten und deren Pose zuverlässig zu bestimmen. Eine Überprüfung der Objektposen mithilfe eines digitalen Zwillings und einer Physiksimulation liefert dabei die Relationen von Objekten mit der Umgebung auf semantischer Ebene. Die Autoren zeigen, dass die Objektposen robust genug sind, sodass ein mobiler Manipulator Tische und Regale aufräumen kann.

Als Gastherausgeber dieser e\&i-Ausgabe möchten wir uns an dieser Stelle ganz herzlich bei den Autor/innen für die Beiträge zum Themenschwerpunkt mobile Manipulation bedanken. Bei dieser Gelegenheit ergeht auch unser Dank an die österreichische Gesellschaft für Mess-, Automatisierungs- und Robotertechnik GMAR (http://www.gmar.at/) für die laufende Unterstützung. Mobile Manipulatoren und ähnlich geartete Robotersysteme sind eine unumgängliche Technologie für die Digitalisierung von Produktionsund Wertschöpfungsprozessen. Mit diesem Sonderheft besteht die Zuversicht, aufzeigen zu können, welche Entwicklungen in Österreich vorangetrieben werden und welche Anwendungspotenziale diese Technologie für Unternehmen eröffnet.

Hinweis des Verlags Der Verlag bleibt in Hinblick auf geografische Zuordnungen und Gebietsbezeichnungen in veröffentlichten Karten und Institutsadressen neutral. 